\title{
A Numerical Analysis of Wind Flow in Urban Areas
}

\author{
Thi Kim Dung Hoang ${ }^{*}$, Phu Khanh Nguyen ${ }^{2}$ \\ ${ }^{1,2}$ Department of Aeronautical and Space Engineering, Hanoi University of Science and Technology, Vietnam \\ *Corresponding author: dung.hoangthikim@hust.edu.vnordunghtk.dase@gmail.com
}

\begin{abstract}
The high population density in Vietnam create many challenges in its urban development and supply of residential housing. To accommodate the large population, the buildings tend to go vertical and high density to maximize the development ratio in the limited land space and in the conservation of natural geomorphology. The high density of building blocks reduces the permeability for wind penetration. In the extreme case, the housing estate becomes a wind-obstructing structure that could be causing to environmental problems and health hazard due to low level of ventilation follow. So, a better understanding about wind flow in urban areas could help resolve some of the main difficulties that a designer might encounter to improve the geometries of urban areas. Therefore, this article performs the simulation methodology using Computational Fluid Dynamics (CFD) tools in ANSYS FLUENT software to carry out the urban wind environment in the simple planning and design urban areas. The studied parameters were wind velocity $10 \mathrm{~m} / \mathrm{s}$ in the case of straight wind and in the case of $45^{\circ}$-cross wind. CFD is an effective and efficient tool to visualize a flow phenomenon of a particular flow problem. The advanced computational technique could calculate the fluid properties at all points defined within the model domain, providing a complete set of data within the model. However, it is important to highlight that CFD technologies have not been completely reliable as model assumptions are still unavoidable for the present computational technologies to resolve turbulent flows. Understanding the limitations of CFD is crucial to reduce numerical errors and realize possible erroneous data in the model.
\end{abstract}

Keywords-Wind Flow, Urban, Vietnam, CFD, ANSYS FLUENT

\section{INTRODUCTION}

$\mathrm{T}_{\mathrm{b}}$ he high population density in Vietnam create many challenges in its urban development and supply of residential housing. The average population density of Vietnam is approximately 305 persons per $\mathrm{km}^{2}$ while less than $33.6 \%$ of total population is urbanized. To accommodate the large population, the buildings tend to go vertical and high density to maximize the development ratio in the limited land space and in the conservation of natural geomorphology.

The urbanization pattern appears to be beneficial to its economic growth and accommodation of its large population, but also to be causing to environmental problems and health hazard due to low level of ventilation follow. The high density of building blocks reduces the permeability for wind penetration. In the extreme case, the housing estate becomes a windobstructing structure. The leeward area would suffer from the lack of ventilation due to the wake effects created by the "wind-obstructing" development.

Recently, many efforts have been made to study the urban wind environment, [1-3]. Blocken et al. [1] studied on wind speed conditions in passages between parallel buildings to carry out the atmospheric boundary layer flow by using Computational Fluid Dynamics (CFD) method. The pedestrian wind environment near tall buildings and interference effect in two and three tall buildings was addressed by Mittal et al. [2]. Effect of the presence of a shorter building in front of a taller building on the wind characteristics and effect of spacing to height ratio on pressure at pedestrian level were performed using CFD tools in ANSYS FLUENT software. They concluded that the combination of interference effect and downwash effect of wind induced the wind pressure increment near pedestrian level of the taller building about three times than that of the shorter building. The velocity in low pressure region between two buildings got amplified by $30 \%$ of average velocity for domain without building because of wind recirculation. Finally, the placement of an adjacent shorter building attached to the taller building reduced the pressure on building by about $65 \%$. It also agreed with the effectiveness of podium in reducing pressure near the tall building. Shishegar [3] remarked that urban climate was influenced by several factors such urban morphology and density, the properties of urban surfaces and vegetation cover. The inappropriate using of these factors could change the microclimate of urban areas. Shishegar summarized that streets geometry and orientation on airflow and solar access in an urban canyon were key factors in providing a pleasant microclimate at pedestrian level in an urban canyon. This study was conducted at wind speed $5 \mathrm{~m} / \mathrm{s}$.

These studies provide comprehensive and in-depth information of the fluid dynamics in various aspects. However, the application of the research findings to complex urban wind environment is limited by the fact that the cases studied are too ideal and significantly deviated from reality which is a mixture of buildings, street canyon and topographical terrain.

This article introduces the simulation methodology using CFD tools in ANSYS FLUENT software applied in the urban wind environment in the simple planning and design urban areas. The studied parameters were 
The Third International Conference on Civil Engineering Research (ICCER)

August $1^{\text {st }}-2^{\text {nd }}$ 2017, Surabaya - Indonesia

wind velocity $10 \mathrm{~m} / \mathrm{s}$ in the case of straight wind and in the case of $45^{\circ}$-cross wind. 


\section{METHOD}

\section{II.1. Urban area model}

Detail of the urban area is designed and presented in Figure 1 in both 2D view and 3D view. The urban has dimensions of $700 * 700 \mathrm{~m}^{2}$ includes about 204 buildings. Each building has $24 \mathrm{~m}$ in width, $22 \mathrm{~m}$ in length and $49.3 \mathrm{~m}$ in height. Interval between two buildings is $15 \mathrm{~m}$. This is early design phase of building projects in urban areas of Hanoi, Vietnam.
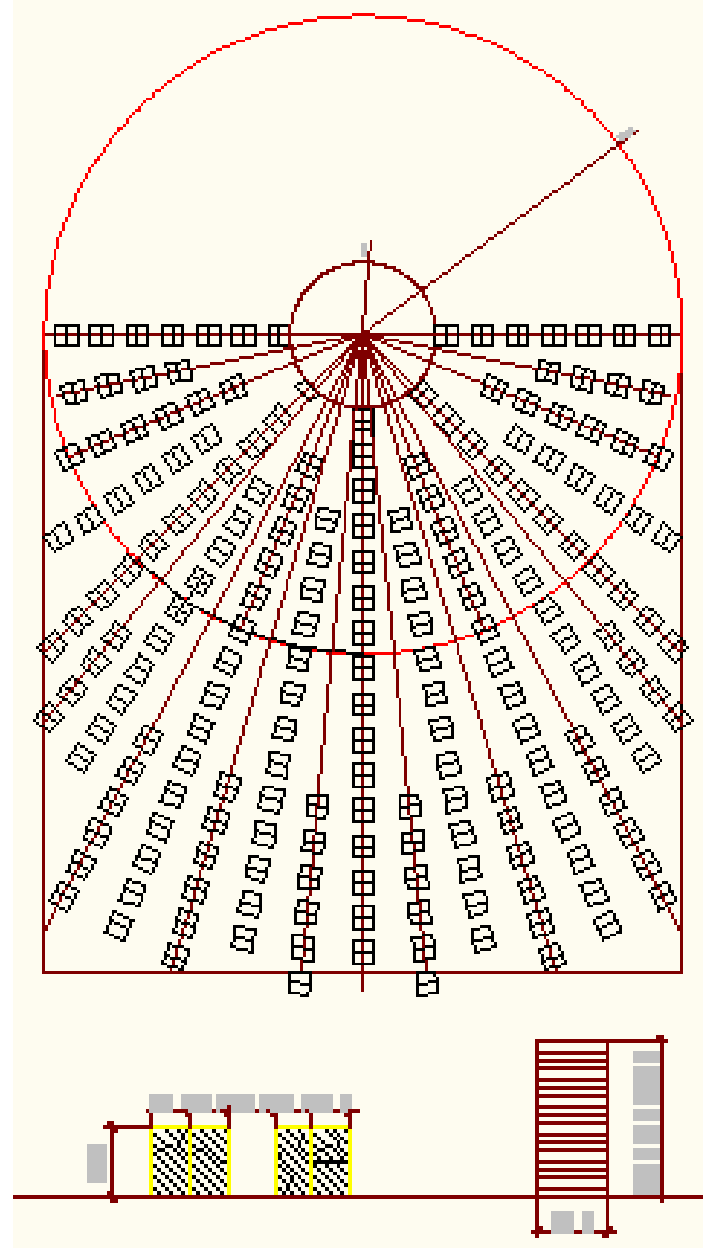

a. $2 \mathrm{D}$ view

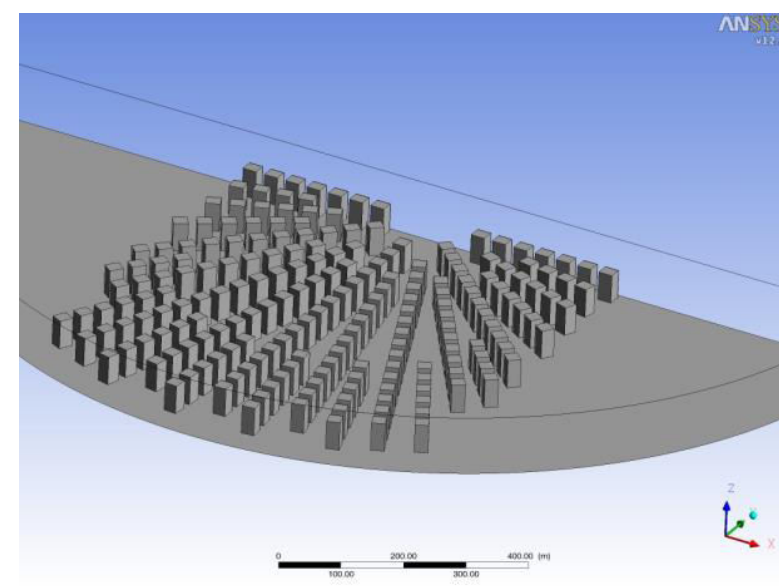

b. 3D view

Figure 1. Urban areas model in CFD

\section{II.2. Numerical setup}

The geometrical model used for the CFD simulation is generated by the ANSYS Design Modeler. The computational domain of urban areas model is identified as shown in Figure 2.

In the next step, meshes is generated from geometrical

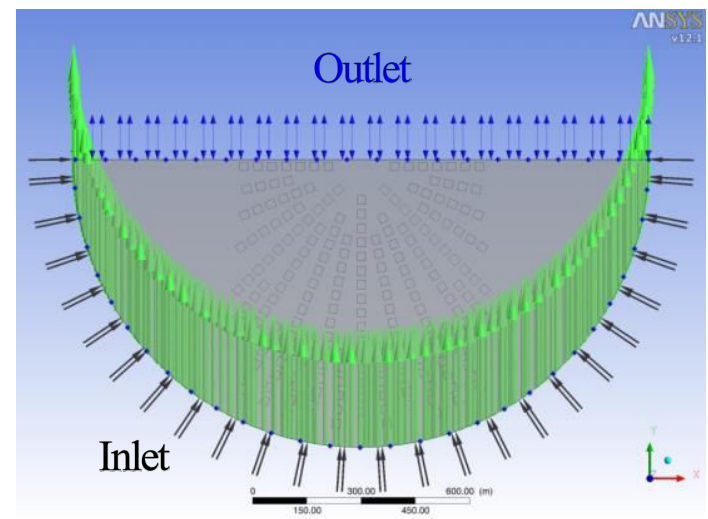

Figure 2. Computational domain and boundary conditions

model by ANSYS Meshing. This computational domain included 16,000 elements with a combination of tetrahedral and prismatic elements.

The turbulent model considered in this study is standard k- $\varepsilon$ model. It is among the best steady-state models that can model the separation flows well, especially for urban level scales. The standard k- $\varepsilon$ model is a semi-empirical model based on model transport equations for the turbulence kinetic energy $(k)$ and its dissipation rate $(\varepsilon)$ [4].

The boundary conditions are showed in figure 2 with detail as follow:

- Velocity inlet: $10 \mathrm{~m} / \mathrm{s}$ in two cases: one is straight wind and other is $45^{\circ}$-cross wind.

- Outlet pressure: ambient pressure.

- Wall: Buildings are identified as solid wall.

\section{RESULTS AND DISCUSSION}

When building a structure it is important to calculate wind load to ensure that the structure can withstand high winds, especially if the building is located in an area known for inclement weather. Following Vietnamese climate, this study is effected at wind speed at level 5 of Beaufort scale corresponding to wind speed $10 \mathrm{~m} / \mathrm{s}$. There are two cases of wind: straight wind and $45^{\circ}$-cross wind. The $45^{\circ}$-cross wind is the wind that inclines an angle of $45^{\circ}$ in the right side of urban areas.

Figure 3 shows pressure distribution of entire urban areas. Figure 4 and Figure 5 present velocity distribution and vector of velocity respectively of four cross-section urban areas at altitude $10 \mathrm{~m}, 20 \mathrm{~m}, 30 \mathrm{~m}$ and $40 \mathrm{~m}$. Finally, Figure 6 presents streamline of wind around building of urban areas at altitude $20 \mathrm{~m}$. 


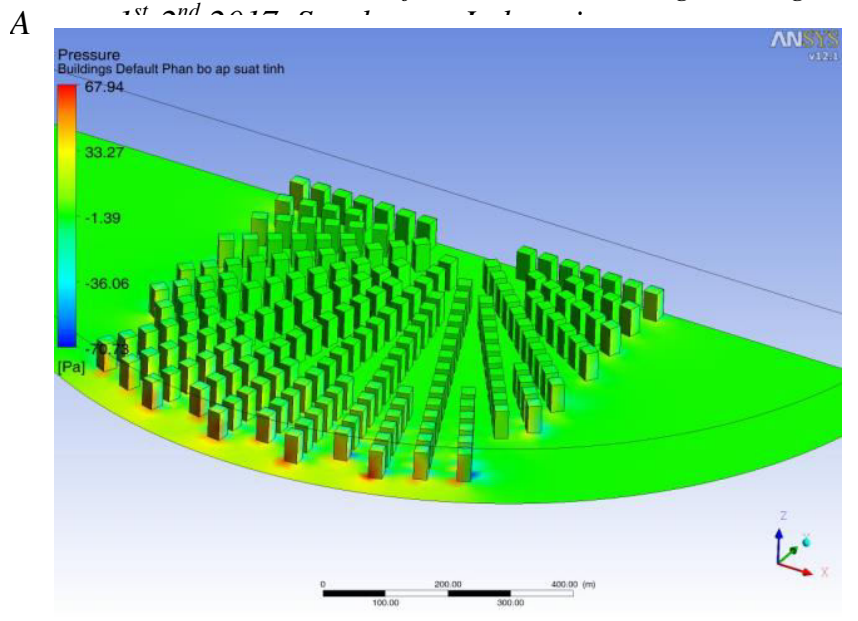

a. Straight wind

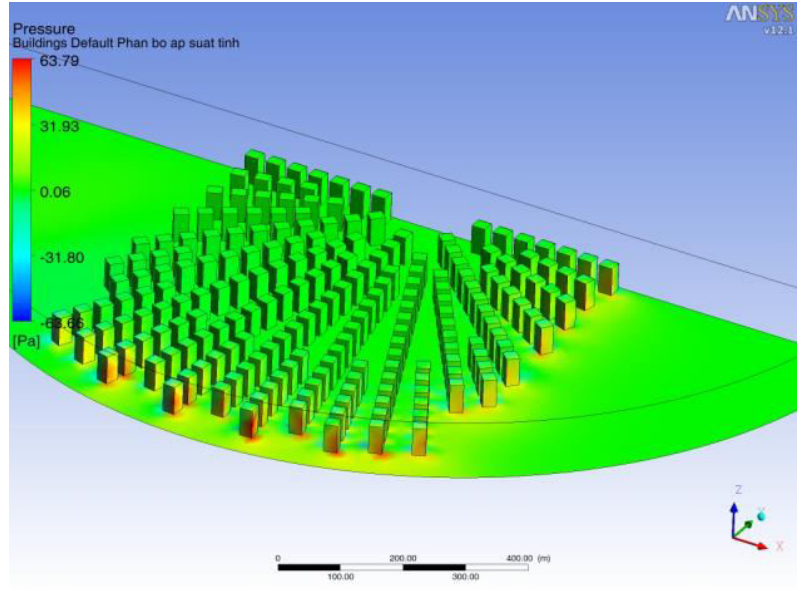

b. $45^{\circ}$-crosswind

Figure 3. Distribution of pressure

The air over urban areas could be divided into main layers: urban canopy layer and urban boundary layer. The urban canopy layer is the layer blow roof tops in the spaces between buildings and influenced by solar energy falling on building facades and ground. The urban boundary layer is above the average height of buildings [3]. The urban boundary layer has a monotone tendency of pressure while urban canopy layer has a complex pressure distribution (Figure 3).

Pressure distribution of urban areas is symmetric for the case of straight wind (Figure 3a) but is dissymmetric for $45^{\circ}$-cross wind (Figure $3 \mathrm{~b}$ ).

At ground level pressure is maximal at impact position where the wind directly attacks buildings. From impact position in the same altitude level, the wind follows the shape of buildings and causes a reduction of pressure at two side of buildings. When altitude level rises up, the same phenomenon of pressure has found out but the level of maximum pressure falls down. Finally, pressure reduces from outside to center of urban areas.

The symmetry of straight wind and dissymmetry of $45^{\circ}$-cross wind has been found clearly in Figure 4 and Figure 5. With increasing of altitude, velocity rises up and vector of velocity becomes more and more stable. Pressure and velocity of straight wind is rather higher than those of $45^{\circ}$-cross wind.

At ground, the characteristics of wind are low wind speed, high pressure. With the orientation of wind to the centre of urban areas, the vortex flow has been found in the centre and behind buildings that located in around $\pm 30-45^{0}$ from the centreline of urban areas (Figure 5a). Vortex flow is also appeared in the case of $45^{\circ}$-cross wind but at position that moved in the left side and return back to the inlet of wind flow (Figure 5b).

For more detail, streamlines of air flow at altitude level $20 \mathrm{~m}$ are described in Figure 6. Air flow behind buildings is complex and creates a vortex.

\section{CONCLUSION}

In conclusion, Computational Fluid Dynamics (CFD) is an effective and efficient tool to visualize a flow phenomenon of a particular flow problem. The advanced computational technique could calculate the fluid properties at all points defined within the model domain, providing a complete set of data within the model. However, it is important to highlight that CFD technologies have not been completely reliable as model assumptions are still unavoidable for the present computational technologies to resolve turbulent flows. Understanding the limitations of CFD is crucial to reduce numerical errors and realize possible erroneous data in the model.

As wind and turbulence within the urban boundary layer determine horizontal and vertical dispersion and transport of air pollutants in towns and is thus important for health of citizens, effects of geometry of buildings, urban areas and properties of wind flow may need to run very large sites for design exploration exercises.

\section{ACKNOWLEDGEMENTS}

The authors would like to thank to ANSYS, Inc. for the authorization of using ANSYS software in simulation works.

\section{REFERENCES}

[1] B. Blocken, J. Carmeliet and T. Stathopoulo, "CFD Evaluation of Wind Speed Conditions in Passages Between Parallel Buildings Effect of Wall-Function Roughness Modifications for The Atmospheric Boundary Layer Flow," Journal of Wind Engineering and Industrial Aerodynamics, 95, 941-962, 2007.

[2] A. K. Mittal, D. Ghosh, S. Behera, I. A. Siddiqui, D. S. Dharmshaktu, "Wind Flow Simulation in The Vicinity of Tall Buildings Through CFD," presented at the Eighth Asia-Pacific Conference on Wind Engineering, Chennai, India, 2013.

[3] N. Shishegar, "Street Design and Urban Microclimate: Analyzing the Effects of Street Geometry and Orientation on Airflow and Solar Access in Urban Canyons," Journal of Clean Energy Technologies, Vol.1, No.1, 52-56, 2013.

[4] D. C. Wilcox, "Turbulence Modeling for CFD", Second edition, Anaheim: DCW Industries, pp. 174, 1998. 
Regional Conference in Civil Engineering (RCCE)

The Third International Conference on Civil Engineering Research (ICCER)

A
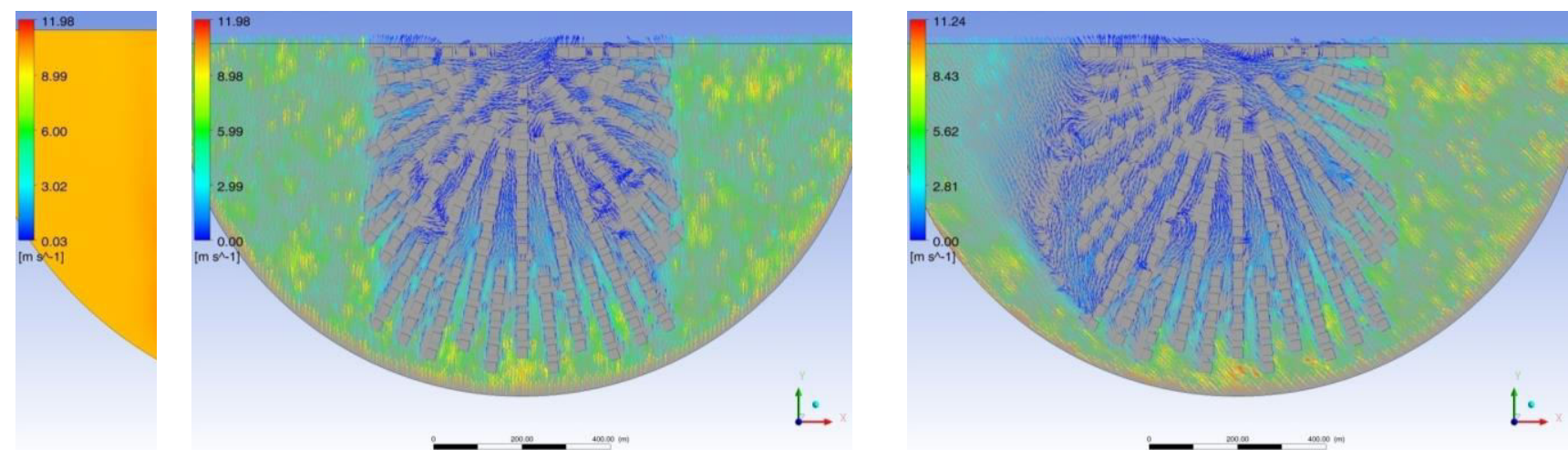

Altitude $10 \mathrm{~m}$
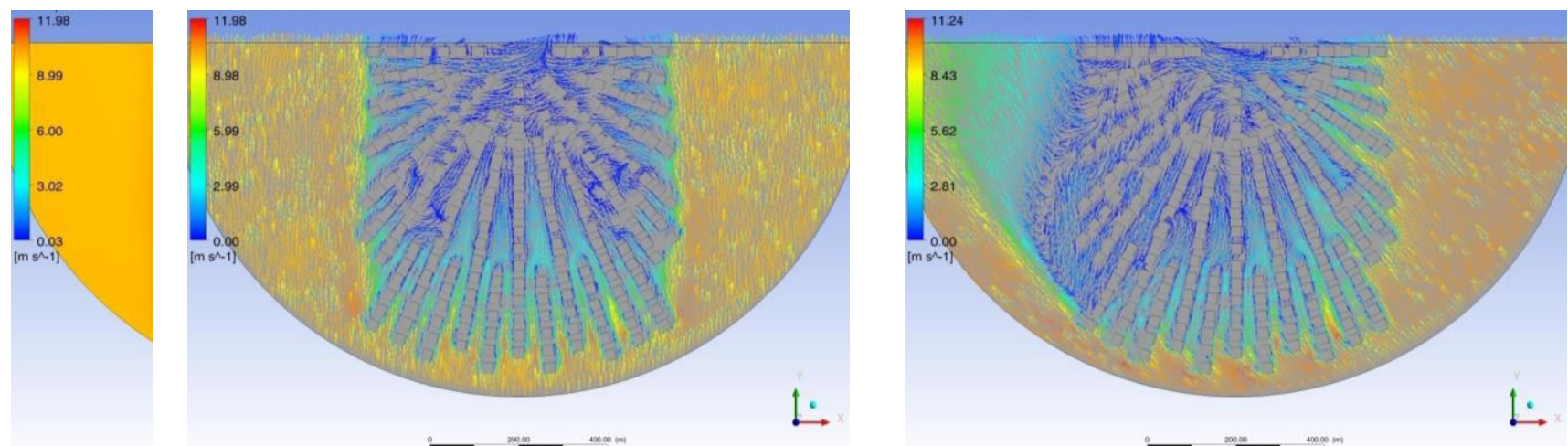

Altitude $20 \mathrm{~m}$
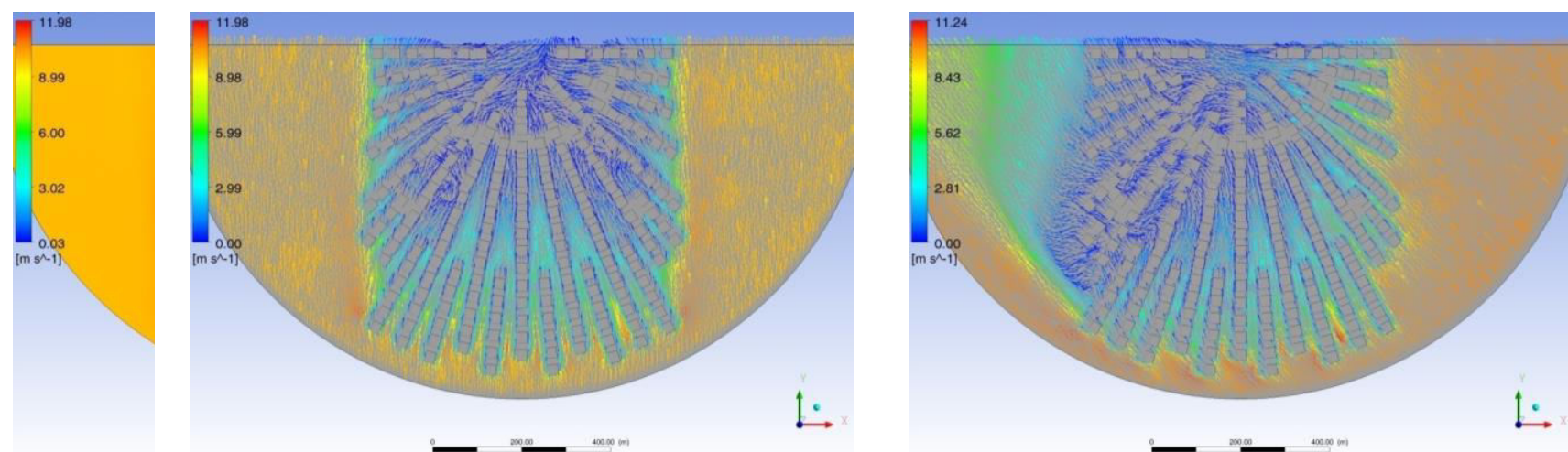

Altitude $30 \mathrm{~m}$

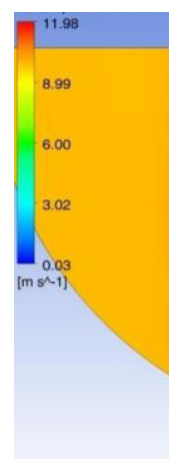

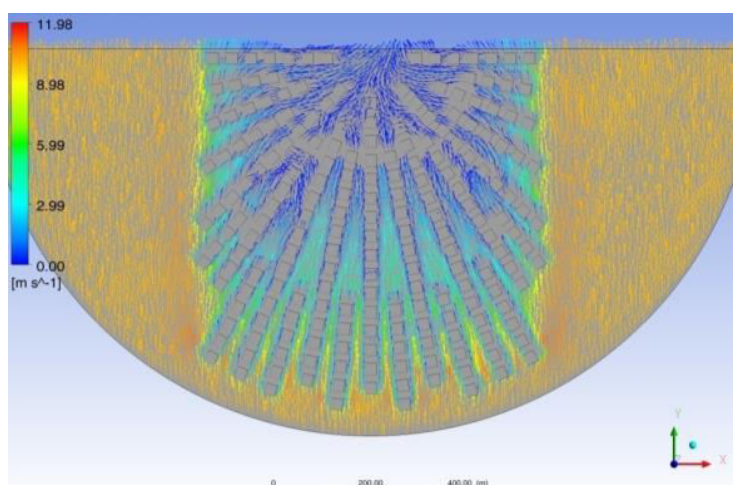

Altitude $40 \mathrm{~m}$

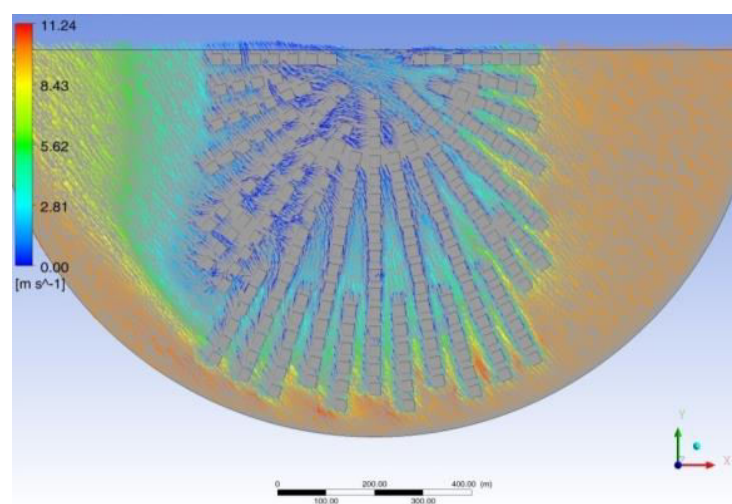

a. Straight wind

b. $45^{\circ}$-cross wind

Figure 5. Vectors of velocity at different altitude level 
Regional Conference in Civil Engineering (RCCE)

The Third International Conference on Civil Engineering Research (ICCER)

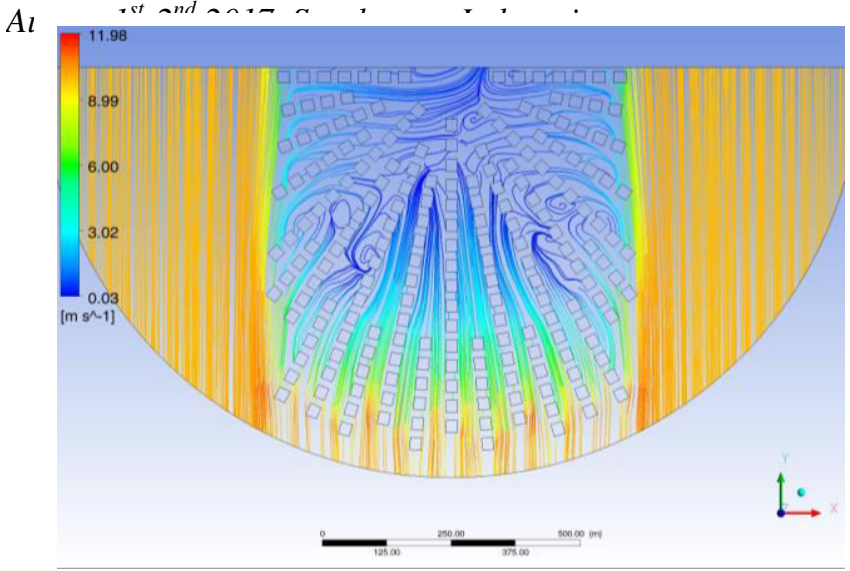

a. Straight wind

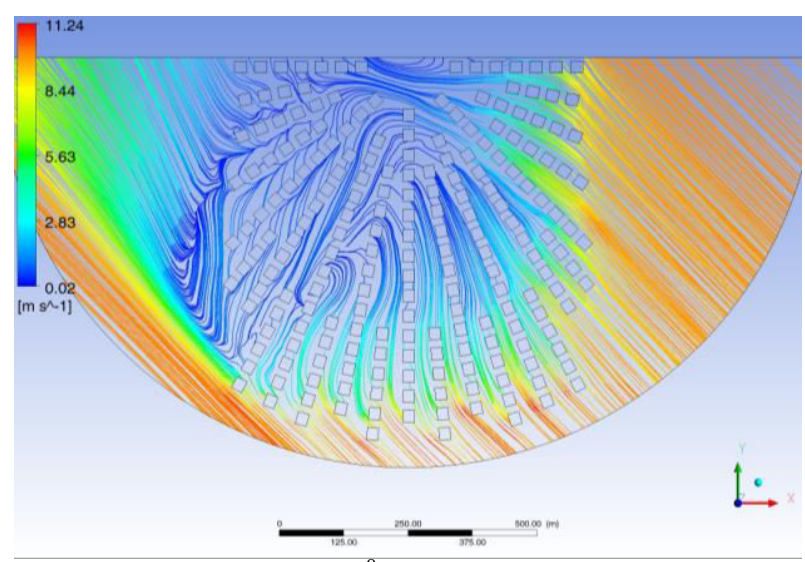

b. $45^{0}$-crosswind

Figure 6. Streamline at altitude $20 \mathrm{~m}$ 